\title{
PICOSECOND X-RAY DIFFRACTION FROM LASER-SHOCKED COPPER AND IRON
}

J. S. Wark, J. F. Belak, G. W. Collins, J. D. Colvin, H. M. Davies, M. Duchaineau, J. H. Eggert, T. C. Germann, J. Hawreliak, A. Higginbotham, B. L. Holian, K. Kadau, D. H. Kalantar, P. S. Lomdahl, H. E. Lorenzana, M. A. Meyers, B. A. Remington, K. Rosolankova, R. E. Rudd, M. S. Schneider, J. Sheppard, J. S. Stolken

September 2, 2005

14th APS Topical Conference on Shock Compression of Condensed Matter Baltimore, MD, United States July 31, 2005 through August 5, 2005 
This document was prepared as an account of work sponsored by an agency of the United States Government. Neither the United States Government nor the University of California nor any of their employees, makes any warranty, express or implied, or assumes any legal liability or responsibility for the accuracy, completeness, or usefulness of any information, apparatus, product, or process disclosed, or represents that its use would not infringe privately owned rights. Reference herein to any specific commercial product, process, or service by trade name, trademark, manufacturer, or otherwise, does not necessarily constitute or imply its endorsement, recommendation, or favoring by the United States Government or the University of California. The views and opinions of authors expressed herein do not necessarily state or reflect those of the United States Government or the University of California, and shall not be used for advertising or product endorsement purposes. 


\title{
PICOSECOND X-RAY DIFFRACTION FROM LASER-SHOCKED COPPER AND IRON
}

\author{
J.S. Wark ${ }^{*}$, J.F. Belak ${ }^{\dagger}$, G.W. Collins ${ }^{\dagger}$, J.D. Colvin ${ }^{\dagger}$, H.M. Davies** ${ }^{*}$, M. Duchaineau ${ }^{\dagger}$, \\ J.H. Eggert ${ }^{\dagger}$, T.C. Germann ${ }^{\ddagger}$, J. Hawreliak* ${ }^{*}$, A. Higginbotham* ${ }^{*}$, B.L. Holian ${ }^{\ddagger}$, K. \\ Kadau $^{\ddagger}$, D.H.Kalantar ${ }^{\dagger}$, P.S. Lomdahl ${ }^{\ddagger}$, H. E. Lorenzana ${ }^{\dagger}$, M.A. Meyers ${ }^{\S}$, B.A. \\ Remington $^{\dagger}$, K. Rosolankova* ${ }^{*}$ R.E. Rudd ${ }^{\dagger}$, M.S. Schneider ${ }^{\S}$, J. Sheppard* and J. S. \\ Stolken ${ }^{\dagger}$
}

\author{
*Department of Physics, Clarendon Laboratory, Parks Road, University of Oxford, OX1 3PU, United \\ Kingdom \\ ${ }^{\dagger}$ Lawrence Livermore National Laboratory, Livermore, CA 94550 \\ ${ }^{* *}$ AWE Aldermaston, Reading, United Kingdom \\ ${ }^{\ddagger}$ Los Alamos National Laboratory, Los Alamos, NM 87545

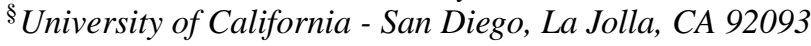

\begin{abstract}
In situ X-ray diffraction allows the determination of the structure of transient states of matter. We have used laser-plasma generated X-rays to study how single crystals of metals (copper and iron) react to uniaxial shock compression. We find that copper, as a face-centred-cubic material, allows rapid generation and motion of dislocations, allowing close to hydrostatic conditions to be achieved on sub-nanosecond timescales. Detailed molecular dynamics calculations provide novel information about the process, and point towards methods whereby the dislocation density might be measured during the passage of the shock wave itself. We also report on recent experiments where we have obtained diffraction images from shockcompressed single-crystal iron. The single crystal sample transforms to the hcp phase above a critical pressure, below which it appears to be uniaxially compressed bcc, with no evidence of plasticity. Above the transition threshold, clear evidence for the hcp phase can be seen in the diffraction images, and via a mechanism that is also consistent with recent multi- million atom molecular dynamics simulations that use the Voter- Chen potential. We believe these data to be of import, in that they constitute the first conclusive in situ evidence of the transformed structure of iron during the passage of a shock wave.
\end{abstract}

Keywords: X-ray diffraction, Phase transitions, Plasticity

PACS: $* * * * * * * * * * * . * *$

\section{INTRODUCTION}

There are many experiments that have been performed over the years in the field of shock wave physics that might justly be described as 'blackbox' physics - a sample is shocked, its response measured, logged, and filed, without any meaningful questions being asked as to what actually happened. Whilst such an approach may well have its place it rarely provides the necessary insight that both satisfies an underlying intellectual curiosity, and also, importantly, leads to a deeper appreciation of the fundamental science upon which true progress is almost invariably predicated. In all but the most simplest situations (which might be considered to be a uniaxial elastic wave along a principal axis in a single crystal with a stress below the asymptotic Hugoniot Elastic Limit (HEL) where all of the atoms within a unit cell 
merely move along the shock propagation direction) the atoms within a shocked solid respond in a complicated manner, yet the question 'what happens at the lattice and atomic level?', although possessing a long history, is one that has only started to be asked in earnest in recent years.

Uniaxial compression above the HEL results in plastic deformation, and in turn such deformation demands both the existence and motion of large numbers of dislocations. Yet it is well known that careful studies of recovered samples consistently conclude that the number of dislocations present after the event are insufficient, often by several orders of magnitude, to explain the observed plastic strain rate. The rate at which plastic strain can occur, $\dot{\varepsilon}$, is governed by the well-known equation attributed to Orowan [1]

$$
\dot{\varepsilon}=N_{\mathrm{m}}|\mathbf{b}| \bar{v}
$$

where $N_{\mathrm{m}}$ is the number of mobile dislocations, $\mathbf{b}$ the Burger's vector of the dislocations, and $\bar{v}$ their mean velocity. As stated above (unless one is prepared to accept the concept of supersonic dislocations) the plastic strain rates observed in real crystalline materials at ultra-high strain rates often demands mobile dislocation densities vastly in excess of the dislocation densities inferred from the study of the microscopic structure of recovered samples. One is inevitably led to conclude that the transient dislocation density during the passage of the shock is considerably higher than that which exists both before and after the shock, yet conclusive experimental proof that this is the case remains illusive. In order to resolve such a fundamental problem we clearly need to develop techniques that allow us to directly measure plastic strain (and strain rate) within a shocked sample and, if possible, yield information on the transient dislocation densities.

An understanding of the detailed mechanisms by which atoms rearrange under shock compression will also be demanded if we are to fully understand shock-induced polymorphic phase transitions. Perhaps the easiest class of such transitions to visualize are the so-called Martensitic transitions, where the atoms can be considered to 'click into place', rather than rearrange via a diffusive mechanism, but even here our understanding of what actually occurs, and on what time-scale, is sketchy, mainly because such transitions are often fully reversible, and thus little information can be gleaned from the recovered sam- ple. The most famous of these transitions is the wellknown $\alpha \rightarrow \varepsilon$ transition in iron, where the initially body centred cubic iron (bcc) is thought to transform to a hexagonal close-packed (hcp) structure. This transition is of particular importance, as, indeed, it was the first to be inferred in a shock-wave measurement over half a century ago (via the observation of a multiple-wave structure).

\section{X-RAY DIFFRACTION}

Time-resolved X-ray diffraction offers the possibility, at least in principle, to answer many of the fundamental questions in the field of shock wave physics outlined briefly above. Not only can transient diffraction give information about the structure of the compressed material, but it can provide some information both about plastic strain and phase transformations, but also, for crystals belonging to certain lattice types, it offers the possibility of providing a direct measurement of in situ dislocation densities.

For a perfect crystal the diffraction of X-rays governed by Bragg's law, where for a plane of Miller indices $(h, k, l)$, the diffraction of monochromatic radiation of wavelength $\lambda$ occurs at an angle $\theta$ to the plane of spacing $d_{h k l}$, where

$$
2 d_{h k l} \sin \theta=n \lambda \text {. }
$$

Compression of the lattice clearly shifts the angle at which diffraction occurs, and for small compressions, the change in diffraction angle is related to the change in the lattice spacing according to a differentiation of Bragg's law:

$$
\frac{\Delta 2 d_{h k l}}{2 d_{h k l}}=-\cot \theta \Delta \theta
$$

It is important to note that the changes in lattice spacings that result in the shift of the diffracted radiation correspond to the elastic components of strain. Indeed, this is why X-ray diffraction is potentially such a useful diagnostic - it can probe the lattice spacing in any direction (at least for thin samples), and thus can provide information about the elastic strain both perpendicular, $\varepsilon_{\perp}^{\mathrm{e}}$ and parallel, $\varepsilon_{\|}^{\mathrm{e}}$, to the shock front. Such a measurement should allow us to extract the plastic strain: we assume that the plastic dilatation is zero:

$$
\varepsilon_{\perp}^{\mathrm{p}}+2 \varepsilon_{\|}^{\mathrm{p}}=0
$$




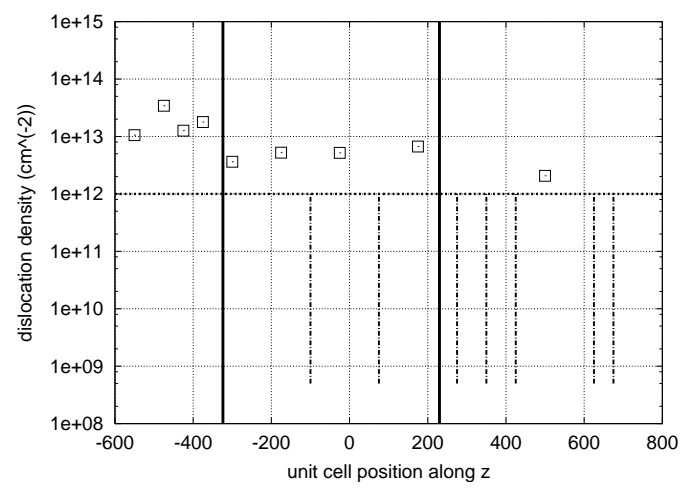

FIGURE 1. Dislocation densities in different regions of the MD simulation inferred from the simulated diffraction peaks. Positions of the prismatic loops are indicated. Density values with too high errors are omitted and replaced by dashed vertical lines representing the range of their possible values. Note the higher value before the first prismatic loop.

where the superscript here denotes plastic strain. Thus, knowing that the strain is uniaxial, i.e. that $\varepsilon_{\|}^{\mathrm{e}}=-\varepsilon_{\|}^{\mathrm{p}}$, we see that not only do measurements of elastic strain parallel to the shock front give direct measurements of the plastic strain parallel to the front, but with the additional information of the elastic strain perpendicular to the front, the plastic strain in this direction is also measured, and can be compared with the elastic strain in this direction.

\section{DISLOCATIONS IN FCC CRYSTALS}

The above discussion has assumed that the Bragg angle is completely determined by the lattice spacing. However, stacking faults in face centred cubic (fcc) crystals can also lead to shifts in the diffraction angle, though these can be more than an order of magnitude smaller than the shifts due to compression. Indeed, this well-known effect was discussed in the classic text by Warren [2], has subsequently been used by metallurgists for many years as a dislocation diagnostic, and was proposed as a method of determining dislocation densities in shocked crystals by Zaretsky. [3] For reasons of space, we will not enter into a full discussion here, and it suffices to say that a stacking fault in an fcc crystal can be thought of as a thin layer of hcp material. Each fault thus introduces a known phase shift between the X-ray and the lattice, and the Bragg peak will shift accordingly by an amount and direction that depends upon the Miller indices and the number of planes between stacking faults. It can be shown that diffraction from the (002) planes shifts by an angle

$$
\Delta(2 \theta)_{002}=-\frac{\sqrt{3} \alpha \tan \theta_{002}}{4 \pi},
$$

where $\alpha$ is the inverse of the mean number of planes between stacking faults. In contrast, for (004) the shift is in the opposite direction:

$$
\Delta(2 \theta)_{004}=+\frac{\sqrt{3} \alpha \tan \theta_{004}}{8 \pi} .
$$

It is thus possible to use eqns (5) and (6) to infer dislocation densities and real compressions from the positions of the diffraction peaks. Conclusive experimental determination of dislocation densities by such a method has yet to be confirmed (though some anomalous results may provide some evidence in this direction [4]) and further experiments in this area are planned. It may be thought that the shock-induced local distortion of the lattice might make such effects impossible to measure. As a preliminary test of this, we decided to post-process the results of a molecular dynamics simulation, producing a simulated diffraction pattern including the (002) and (004) planes, and used the centers of gravity of the diffraction peaks to determine dislocation densities. [5] These dislocation densities inferred from the simulated diffraction measurements were then compared with measurements obtained by producing images of the atoms, with each atom color-coded according to its number of nearest neighbors. Such an image provides a simple means of manually counting the dislocation density. Good agreement between the two methods provides confidence that the X-ray diagnostic may work in determining dislocation densities as ultrahigh strain rates. Full details of this work will be presented in a forthcoming publication, and thus we simply present the main results here.

We generated simulated X-ray diffraction patterns from the atomic positions provided by an MD simulation of a shocked single copper crystal containing $256 \times 10^{6}$ atoms. Its dimensions prior to shock launch were $200 \times 200 \times 1600$ unit cells, with the long side of the sample along the shock propagation direction [001], and the unperturbed unit cell size 


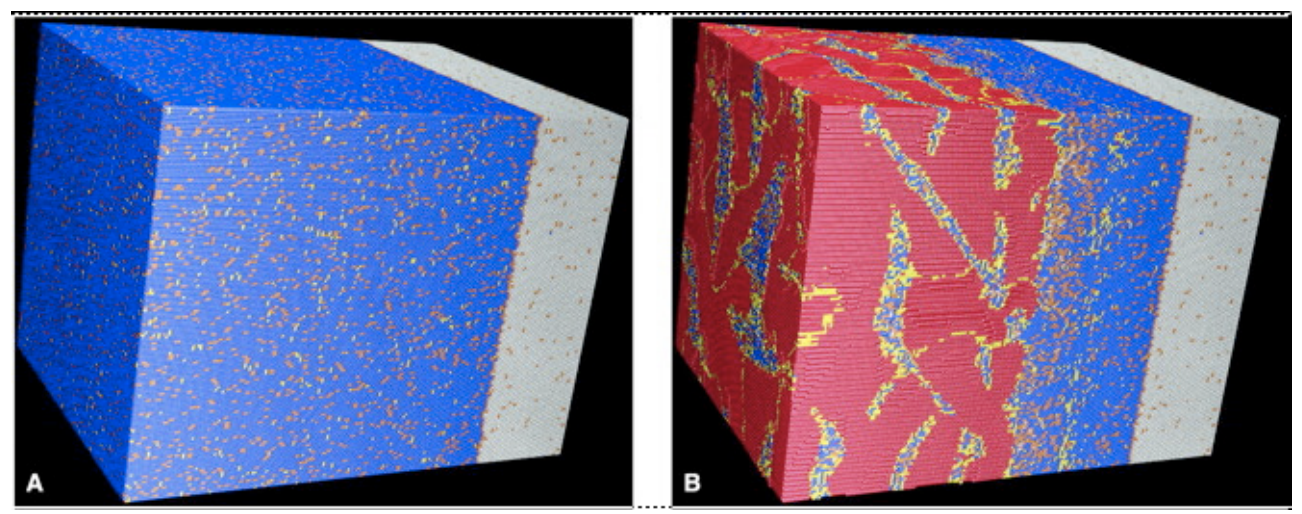

FIGURE 2. MD simulations of shock-compressed single crystal Iron [14]. $u_{p}$ increases between $\mathbf{A}$ and $\mathbf{B}$. Colour legend: grey $=$ unshocked BCC $(\alpha$ phase $)$; blue = uniaxially compressed BCC; red = transformed $\varepsilon$ phase $($ quasi-HCP $)$; yellow = grain boundaries (e.g. twin boundaries).

$a_{0}=3.615 \AA$. The simulation thus spanned $\sim 0.6 \mu \mathrm{m}$ in length. The shock wave launched in the crystal had a $50 \mathrm{ps}$ long linear ramp. The peak pressure generated was $35 \mathrm{GPa}$, thus exceeding the threshold for the onset of homogeneous, as well as inhomogeneous dislocation generation $(\sim 32$ and $\sim 10 \mathrm{GPa}$, respectively [6]). The total duration of the simulation was $\sim 130 \mathrm{ps}$. The long duration of the simulation allowed the observation of relaxation of the shear stress (observed as shear strain) in the diffraction signal, something not observed with shorter simulation times. [9][10]. Two prismatic loops were incorporated into the crystal prior to shock launch. The first one was located at $\sim 0.18 \mu \mathrm{m}$ and the second one at $\sim 0.4 \mu \mathrm{m}$ along the $z$ axis. Further details of the simulation are provided elsewhere, [7] and much was learnt about the role and interplay between ramped compression waves and dislocation sources. However, for the present discussion it suffices to say that large numbers of homogeneously-generated dislocations $\left(>1-2 \times 10^{13} \mathrm{~cm}^{-2}\right)$ were found in the first part of the crystal before the first prismatic loop. The interplay between the ramp and the loop resulted in subsequent regions of the crystal having present dislocations born from the loop, with lower overall densities (by about a factor of 3), where the above numbers are inferred from direct counting of the dislocations. However, importantly, the regions of lower dislocation density resulted in slightly greater relaxation of the shear stress: the very high densities generated homogeneously tended to produce networks of dislocations that 'tangled', and thus had lower velocities, and, via Orowan's equation, were less efficient at relaxing shear stress.

As can be seen from Fig. 1, the dislocation densities inferred from the Fourier Transform of the atomic positions (i.e. the simulated diffraction pattern, and the resultant shift in the diffraction peaks), show excellent agreement with the dislocation densities inferred by direct counting. From the estimated error bars we believe that it should be possible to use time-resolved diffraction techniques to observe the high-dislocation densities that need to exist at ultra-high strain rates. High strain-rate phenomena have been observed via diffraction, with single crystals of copper showing close to hydrostatic behavior on sub-nanosecond time-scales, [8] and dislocation densities of the same order as those present in this MD simulation are expected in such experiments, depending upon how well simulated dislocation velocities match those actually present. Furthermore, the laser-plasma technique used in these experiments allows for the generation of X-rays of multiple wavelengths, and also the experimental set-up is such that the shocked-crystal subjects a relatively large solidangle to the X-ray source. It should thus be possible to record diffraction from (002) and (004) planes simultaneously as required.

\section{PHASE TRANSITION IN IRON}

The $\alpha \rightarrow \varepsilon$ phase transition in iron is perhaps the most famous of all shock-induced transitions, and it 
A: 2-D FT (110) plane

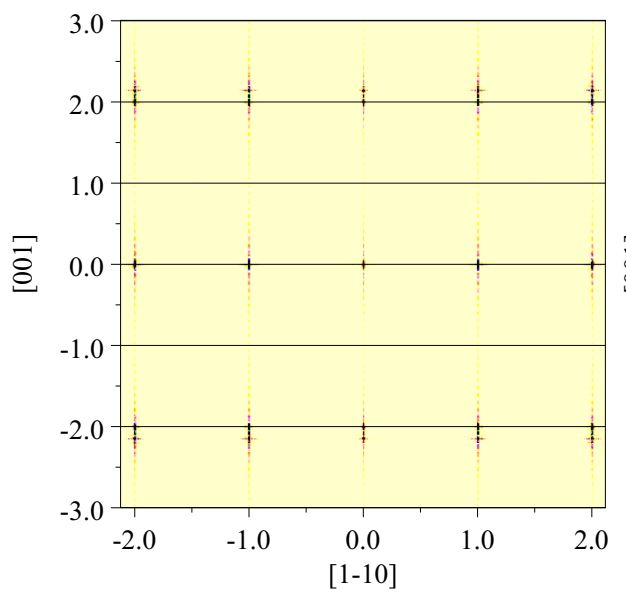

B: 2-D FT (110) plane

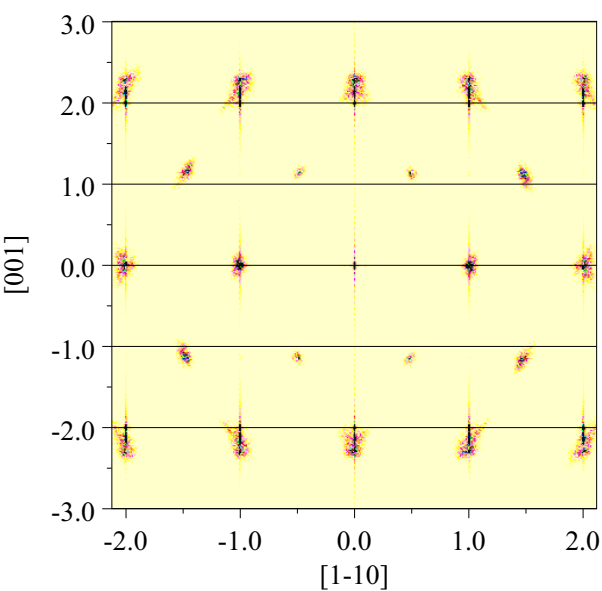

FIGURE 3. 2-D diffraction from (110) BCC planes in Iron MD simulations shown in Fig. 2. Both [11̄0] and [001] vectors are normalised. Elastically compressed simulation $\mathbf{A}$ does not display a hexagonal inverse lattice, whilst simulation $\mathbf{B}$ shows a clear hexagonal basis in the original BCC (110) plane, as well as unshocked, and elastically-compressed bcc phase.

plays a pivotal role in the development of the field of shock wave physics. The first tentative evidence for the transition under shock conditions came half a century ago, when J.M. Walsh reported data on the propagation of high-pressure shock waves in Armco iron that were in disagreement with an extrapolation of Bridgman's original static compressibility measurements. [11] Soon after, Minshall and co-workers resolved the discrepancy, when they found that multiple shock waves propagated within the iron sample. [12] They identified these different wave structures with different phases present in the crystal, and inferred the existence of a polymorphic phase transition at a pressure of $130 \mathrm{kbar}$. It is interesting to note that at the time of these shock experiments, this was an unknown transition, and it was several years after Minshall's shock-wave work that the crystallographic evidence for a transition at this pressure was obtained in static X-ray diffraction experiments by Jamieson and Lawson. [13] It has been assumed ever since that the transition associated with the multiplewaves seen in shock experiments, and that observed under static high-pressure conditions, are one and the same transition. This discovery of one of the most important phase transitions known to man has long been hailed as one of the major triumphs of the field of shock-wave physics. That said, it is important to recall that to date no conclusive observation of the new structure (i.e. detailed in situ X-ray diffrac- tion evidence) during the passage of the shock itself has been reported until recently. Details of this experimental observation of the phase transition with nanosecond diffraction are presented elsewhere. [15]

The experimental data is in excellent agreement with the main features of MD simulations performed by Kadau and co-workers. [14] Data from a sub-set of these simulations are shown in Fig. 2. Note that below the threshold pressure (image $\mathbf{A}$ ), the bcc lattice is simply elastically compressed. Unlike with simulations of fcc materials, no dislocation generation is observed. Above the transition pressure (image B) a two-wave structure is seen, with an initial wave corresponding to compressed bcc, and then, within a few lattice spacings, the material transforms to the hcp phase. The mechanism of the martensitic transformation corresponds to the [110] (or, degenerately, the [1-10]) plane of the original bcc lattice becoming the c-axis of the hcp phase. On these timescales there is negligible movement of the atoms in a direction normal to the shock propagation direction.

Confirmation of the new phase can be seen in the simulated diffraction patterns presented in Fig 3 , which shows the 2-D Fourier transform in the original [110] plane - i.e. the plane expected to become the c-axis for half of the hcp material . Note in the first diffraction image, corresponding to image $\mathbf{A}$ of Fig. 2 that the diffraction pattern corresponds to the reciprocal lattice of bcc material. In- 
deed, both uncompressed and compressed material can be seen (note two diffraction peaks for those points corresponding to having components of the reciprocal lattice vector parallel to the shock propagation direction - i.e. the vertical axis). In contrast, the second diffraction pattern, corresponding to image $\mathbf{B}$, clearly shows period-doubling, and the new hexagonal structure. Note that there is negligible shift in the diffraction peaks perpendicular to the shock propagation direction, and that this diffraction pattern comprises diffraction from unshocked bcc, elastically compressed bcc, and hcp material (note the three peaks now present around the original bcc diffraction points). These diffraction simulations are in excellent overall agreement with recent experimental data, confirming the rapid nature of the $\alpha \rightarrow \varepsilon$ transition.

\section{SUMMARY}

Time-resolved X-ray diffraction from shockcompressed crystals is a burgeoning area of study, as the technique affords the possibility to directly observe phase transformations in shocked samples, as well as obtain direct information on elastic (and hence, under uniaxial conditions, plastic) strain. Further shifts in rocking curve peaks may in principle be used to directly measure dislocation densities in fcc crystals, and post-processing of atomic positions provided by MD simulations confirms the viability of this technique, at least for very high dislocation densities that might be expected at ultra-high strain-rates.

\section{ACKNOWLEDGMENTS}

This work was conducted under the auspices of the US DOE by the UC Lawrence Livermore National Laboratory and Los Alamos National Laboratory under Contract No. W-7405-Eng-48. Experiments were conducted at the University of Rochester Laboratory for Laser Energetics under the NLUF grants program. Additional support was provided by the Department of Energy under Grants DEFG0398DP00212 and DEFG0300SF2202, by the UK EPSRC under grant GR/R25699/01, by the Advanced Simulation and Computing Materials and
Physics Modeling program and LDRD programs at LANL and LLNL.

\section{REFERENCES}

1. Orowan, E., Proc. Phys. Soc., 52, 8-22 (1940).

2. Warren, B. E., X-ray Diffraction, Dover Publications, Inc., 1969.

3. Zaretsky, E., J Appl Phys, 78, 3740-7 (1995)

4. AlÕtshuler, L. V., Egorov, L. A., Nitochkina, E. V., and Orekin, Y. K., Sov. Phys. JETP, 54, 359 (1981).

5. Rosolankova, K., Picosecond X-ray Diffraction from Shock-Compressed Metals: Experiments and Computational Analysis of Molecular Dynamics Simulations, Ph.D. thesis, University of Oxford (2005).

14. Kadau, K., Germann, T. C., Lomdahl, P. S., and Holian, B. L., Science, 296, 1681-1684 (2002).

6. Bringa, E. M., Wirth, B. D., Caturla, M. J., Stolken, J., and Kalantar, D., Nuc. Inst. and Methods Phys. Res. B, 202, 56 (2003).

9. Rosolankova, K., Kalantar, D. H., Belak, J. F., Bringa, E. M., Caturla, M. J., Hawreliak, J., Holian, B. L., Kadau, K., Lomdahl, P. S., Germann, T. C., Ravelo, R., Sheppard, J., and Wark, J. S., "X-Ray Diffraction from Shocked Crystals: Experiments and Predictions of Molecular Dynamics Simulations," in Shock Compression of Condensed Matter, AIP, 2003, pp. 1195-8.

10. Holian, B. L., and Lomdahl, P. S., Science, 280, 2085-2088 (1998).

7. Bringa, E. M., Duchaineau, M., Rosolankova, K., Rudd, R. E., Kalantar, D. H., Remington, B. A., Wark, J. S., and Belak, J., Nature, submitted (2005).

8. Loveridge-Smith, A., Allen, A. M., Belak, J., Boehly, T., Hauer, A., Holian, B., Kalantar, D., Kyrala, G., Lee, R. W., Lomdahl, P., Meyers, M. A., Paisley, D. Pollaine, S., Remington, B., Swift, D. C., Weber, S., and Wark, J. S., Phys. Rev. Lett., 86, 2349-2352 (2001).

11. Walsh, J. M., Bull Am Phys Soc, 29, 28 (1954).

12. Bancroft, D., Patterson, E. L., and Minshall, S., $J$ Appl Phys, 27, 291 (1956).

13. Jamieson, J. C., and Lawson, A. W., J Appl Phys, 33, 776 (1962).

15. Kalantar, D. H., Belak, J. F., Collins, G. W., Colvin, J. D., Davies, H. M., Eggert, J. H., Germann, T. C., Hawreliak, J., Holian, B. L., Kadau, K., Lomdahl, P. S., Lorenzana, H. E., Meyers, M. A., Rosolankova, K., Schneider, M. S., Sheppard, J., Stolken, J. S., and Wark, J. S., Physical Review Letters, to be published (2005). 